\title{
Breathing disorders in congestive heart failure: gender, etiology and mortality
}

\author{
R.S. Silva ${ }^{1,3}$, A.C. Figueiredo ${ }^{1}$, C. Mady² and G. Lorenzi-Filho ${ }^{1}$ \\ ${ }^{1}$ Laboratório do Sono, ${ }^{2}$ Unidade de Insuficiência Cardíaca, Instituto do Coração, Hospital das Clínicas, \\ Faculdade de Medicina, Universidade de São Paulo, São Paulo, SP, Brasil \\ ${ }^{3}$ Disciplina de Medicina e Biologia do Sono, Departamento de Psicobiologia, Escola Paulista de \\ Medicina, Universidade Federal de São Paulo, São Paulo, SP, Brasil \\ Correspondence to: G. Lorenzi-Filho, Laboratório do Sono, Divisão de Pneumologia, Instituto do \\ Coração, Av. Dr. Eneias de Carvalho Aguiar, 44, 05403-900 São Paulo, SP, Brasil \\ Fax: +55-11-3069-5643. E-mail: geraldo.lorenzi@incor.usp.br
}

\begin{abstract}
We investigated the relationship between sleep-disordered breathing (SDB) and Cheyne-Stokes respiration (CSR) while awake as well as mortality. Eighty-nine consecutive outpatients (29 females) with congestive heart failure (CHF; left ventricular ejection fraction, LVEF <45\%) were prospectively evaluated. The presence of SDB and of CSR while awake before sleep onset was investigated by polysomnography. SDB prevalence was 81 and $56 \%$, using apnea-hypopnea index cutoffs $>5$ and $>15$, respectively. CHF etiologies were similar according to the prevalence of SDB and sleep pattern. Males and females were similar in age, body mass index, and LVEF. Males presented more SDB $(P=0.01)$, higher apnea-hypopnea index $(P=0.04)$, more light sleep (stages 1 and 2; $P<0.05)$, and less deep sleep $(P<0.001)$ than females. During follow-up $(25 \pm 10$ months $), 27 \%$ of the population died. Non-survivors had lower LVEF $(P=0.01)$, worse New York Heart Association (NYHA) functional classification $(P=0.03)$, and higher CSR while awake $(P<0.001)$ than survivors. As determined by Cox proportional model, NYHA class IV $(R R=3.95,95 \% \mathrm{Cl}=1.37-11.38, P=0.011)$ and $C S R$ while awake with a marginal significance $(R R=2.96,95 \% \mathrm{Cl}=0.94-9.33$, $\mathrm{P}=0.064$ ) were associated with mortality. In conclusion, the prevalence of SDB and sleep pattern of patients with Chagas' disease were similar to that of patients with CHF due to other etiologies. Males presented more frequent and more severe SDB and worse sleep quality than females. The presence of CSR while awake, but not during sleep, may be associated with a poor prognosis in patients with $\mathrm{CHF}$.
\end{abstract}

Key words: Sleep apnea; Cheyne-Stokes respiration; Congestive heart failure; Gender; Chagas' disease

Publication supported by FAPESP.

Received July 24, 2007. Accepted January 25, 2008

\section{Introduction}

Congestive heart failure (CHF) is a highly prevalent disorder with considerable morbidity and mortality rates despite advancements in the diagnosis and management of patients (1). Although the lifetime risk for developing $\mathrm{CHF}$, regardless of etiology, is 1 in 5 among both men and women (2), little is known about risk factors for the development of CHF in women, perhaps because of the underrepresentation of women in cardiovascular clinical trials (3). Sleep-disordered breathing (SDB) is common in males with $\mathrm{CHF}$ and may be associated with a poor prognosis (47). However, most studies have focused on males and there are fewer data about the characteristics of sleep in women with $\mathrm{CHF}(8,9)$. In addition, there is no information about sleep in patients with CHF due to Chagas' disease, a neglected disease with profound cardiac consequences which affects approximately 18 million people worldwide (10).

SDB in patients with CHF manifests mainly as CheyneStokes respiration (CSR) and also as obstructive sleep apnea (OSA) $(8,9)$. CSR is characterized by recurrent episodes of central apnea, alternating with hyperpnea, during which there is an crescendo-decrescendo pattern of tidal volume (11). CSR pattern is not restricted to sleep but may occur during wakefulness $(12,13)$ as well as 
during exercise (14-16). SDB is associated with recurrent hypoxia and arousals from sleep, which may contribute to increased mortality (17). However, studies of the role of SDB in the prognosis of patients with CHF have provided conflicting results (4-9,11-19). Several studies did not monitor sleep and relied on limited unattended overnight cardiorespiratory polygraphy, making it impossible to determine the contribution of awake CSR to their results $(5,9,16)$. In contrast to sleep, the CSR pattern during wakefulness and exercise has been more consistently associated with a poor prognosis (14-16).

The purpose of the present study was to extend our knowledge by evaluating consecutive CHF patients from a single clinic by full polysomnography (PSG) in order to describe: 1) the presence of SDB and characteristics of sleep according to etiology, including patients with Chagas' disease, 2) the presence of SDB and the characteristics of sleep in men and women, and 3) the relationship between patterns of SDB and CSR while awake with mortality.

\section{Patients and Methods}

\section{Patient selection}

Consecutive patients with $\mathrm{CHF}$ were recruited from the outpatient Heart Failure Unit of the Heart Institute (Instituto do Coração, Hospital das Clínicas, Faculdade de Medicina, Universidade de São Paulo). Patients were eligible if they had 1) resting left ventricular ejection fraction (LVEF) of less than $45 \%$ as determined by echocardiography, 2 ) stable clinical condition as defined by no changes in drug therapy for at least 1 month before evaluation, and 3) age between 18 and 75 years. Patients with the following conditions were excluded: neurological, pulmonary, renal, and musculoskeletal disease, unstable angina, primary valvular heart disease, or a previous diagnosis of SDB. The protocol was approved by the Ethics Committee of our institution and written informed consent was obtained from each patient.

\section{Sleep study}

Overnight PSG was performed using an EMBLA digital system (17 channels, EMBLA Medicare, USA). The following variables were monitored: electroencephalogram (four channels: C3-A2, C4-A1, O1-A2, O2-A1), electrooculogram (two channels: LOC-A2, ROC-A1), electromyogram (two channels: submental and anterior tibialis muscles) using surface electrodes, and electrocardiogram (one channel). Snoring and body position were detected with EMBLA sensors. Airflow was detected with a thermocouple and a pressure flow transducer. Chest and abdominal piezo sensors monitored respiratory effort. Arterial oxygen saturation $\left(\mathrm{SaO}_{2}\right)$ and pulse were recorded with a pulse oximeter.

All PSGs were performed and scored on the basis of guidelines for sleep studies (20). Arousals were defined according to the report of the Sleep Disorders Atlas Task Force of the American Sleep Disorders Association (21). Apneas, hypopneas, and CSR were defined according to the report of the American Academy of Sleep Medicine Task Force (22). Apnea was defined as cessation (>90\% in amplitude) of airflow for at least $10 \mathrm{~s}$. Obstructive apnea was defined as the absence of airflow in the presence of respiratory effort. Central apnea was defined as the absence of airflow with absence of respiratory effort. Hypopnea was defined as a clear reduction ( $>50 \%$ in amplitude) of airflow lasting $10 \mathrm{~s}$ or more and associated with a drop in arterial oxyhemoglobin saturation and/or arousal of at least $3 \%$. CSR was defined by the presence of at least three consecutive central apneas or hypopneas interposed by a crescendo-decrescendo pattern of tidal volume. The apnea-hypopnea index (AHI) was defined by the number of apneas and hypopneas per hour of sleep (22). We defined presence of SDB when the $\mathrm{AHI}$ was above 15 events/h according to several published reports $(23,24)$. CSR was established when at least $80 \%$ of the respiratory events were central in nature, interposed by a crescendodecrescendo pattern of tidal volume, on the basis of $\mathrm{AHI}$ $>15 / h$.

\section{Awake measurements}

Arterial blood gases. Arterial blood samples were drawn $30 \mathrm{~min}$ to $1 \mathrm{~h}$ before the onset of sleep recording, while patients were sitting and awake, after at least $10 \mathrm{~min}$ of undisturbed rest, on room air.

Daytime sleepiness. Daytime sleepiness was assessed by a subjective rating (Epworth Sleepiness Scale, 25) in each patient before the overnight sleep study.

Respiratory pattern. The respiratory pattern was recorded for 10 min while awake in supine position immediately before the lights were turned off during PSG. CSR while awake was defined using the same criteria used during sleep (22).

\section{Follow-up}

All patients were followed regularly at the outpatient clinic. The last follow-up information was obtained by means of a telephone interview with the patient or with his/her family. In the case of a deceased patient, mortality information, including the date of death, was obtained from the next of kin.

\section{Statistical analysis}

All descriptive data are reported as means \pm SD. Differ- 
ences between groups according to respiratory pattern (CSR, OSA, and no SDB) were analyzed by one-way ANOVA. Proportions were compared by the $Z$ test. Gender characteristics were compared by the Student unpaired $t$ test or $Z$ test when appropriate. Survival was estimated by the Kaplan-Meier test and differences between survival curves were determined by the log-rank test. A Cox proportional model, including all variables with a significant level $<20 \%$, was applied. A P value $<0.05$ was considered significant.

\section{Results}

A total of 89 patients (60 men) were enrolled in the study. Eighty-one percent of the patients were on diuretics, $75 \%$ on digitalis, $90 \%$ on vasodilators, and $19 \%$ on ß-blockers. According to the New York Heart Association (NYHA) functional classification, $60 \%$ of the patients were class II, $29 \%$ were class III, and $11 \%$ were class IV. The etiology of CHF was as follows: idiopathic (54 patients), ischemic (11 patients), and Chagas' disease (24 patients). The overall prevalence of SDB in the entire group evaluated was $81 \%$, using an $\mathrm{AHI}>5$ cutoff, and $56 \%$, using an $\mathrm{AHI}>15$ cutoff. Using an $\mathrm{AHI}>15$ cutoff, CSR and OSA were present in 42 and $11 \%$ of patients with idiopathic etiology, 73 and $0 \%$ of those with ischemic etiology, and 44 and $12 \%$ of those with Chagas' disease etiology, respectively. The demographic, clinical and laboratory characteristics of the whole group are divided according to Chagas' disease and remaining etiologies (non-Chagas' disease) are summarized in Table 1. There were no significant differences in any of the characteristics analyzed. The PSG characteristics were similar between patients with Chagas' disease and non-Chagas' disease (Table 2). Moreover, we did not find differences in the presence of SDB among CHF etiologies $(P=0.4)$.

Males and females were similar with respect to age, body mass index, LVEF, as well as arterial blood gases (Table 3). The PSG characteristics according to gender are summarized in Table 4. Females had a better sleep pattern, with signifi- cantly higher total sleep time $(P=0.03)$, increased sleep efficiency $(P=0.03)$, less light sleep (stage $1(P=0.01)$ and stage $2(P=0.02)$ ), and increased deep sleep (stages $3+$ $4(P<0.001))$. In addition, the prevalence of SDB $(P=0.01)$ and $\mathrm{AHI}(\mathrm{P}=0.03)$ was significantly lower in females than in males. Males had higher daytime sleepiness than females $(P=0.04)$.

Respiratory pattern while awake was determined in 75 patients (84\%). The remaining 14 patients had less than 10 min of respiratory recordings while awake due to either micro-sleep or movement artifacts. CSR while awake was
Table 1. Demographic, clinical, and laboratory data of patients with congestive heart failure according to etiology due to Chagas' and non-Chagas' disease.

\begin{tabular}{lccc}
\hline & $\begin{array}{c}\text { Total } \\
\mathrm{N}=89(100 \%)\end{array}$ & $\begin{array}{c}\text { Chagas' disease } \\
\mathrm{N}=24(27 \%)\end{array}$ & $\begin{array}{c}\text { Non-Chagas' disease } \\
\mathrm{N}=65(73 \%)\end{array}$ \\
\hline Age (years) & $58 \pm 12$ & $58 \pm 9$ & $58 \pm 12$ \\
$\mathrm{BMI}\left(\mathrm{kg} / \mathrm{m}^{2}\right)$ & $26 \pm 6$ & $25 \pm 5$ & $26 \pm 5$ \\
$\mathrm{SBP}(\mathrm{mmHg})$ & $121 \pm 26$ & $115 \pm 32$ & $123 \pm 23$ \\
$\mathrm{DBP}(\mathrm{mmHg})$ & $78 \pm 13$ & $78 \pm 12$ & $78 \pm 13$ \\
$\mathrm{LVEF}(\%)$ & $35 \pm 6$ & $38 \pm 5$ & $35 \pm 6$ \\
Awake arterial blood gases & $7.37 \pm 0.3$ & $7.42 \pm 0.03$ & $7.37 \pm 0.4$ \\
$\quad$ Arterial pH & $37 \pm 5$ & $37 \pm 5$ & $37 \pm 5$ \\
$\mathrm{PaCO}_{2}(\mathrm{mmHg})$ & $80 \pm 9$ & $79 \pm 8$ & $80 \pm 10$ \\
$\mathrm{PaO}_{2}(\mathrm{mmHg})$ & & &
\end{tabular}

Data are reported as mean \pm SD. $B M I=$ body mass index; SBP = systolic blood pressure; $\mathrm{DBP}=$ diastolic blood pressure; $\mathrm{LVEF}=$ left ventricular ejection fraction; $\mathrm{PaCO}_{2}=$ arterial carbon dioxide tension; $\mathrm{PaO}_{2}=$ arterial oxygen tension. There were no significant differences between groups (Student unpaired $t$-test).

Table 2. Polysomnographic characteristics of patients with congestive heart failure (CHF) with CHF etiology due to Chagas' disease or not.

\begin{tabular}{lcc}
\hline & Chagas' disease & Non-Chagas' disease \\
\hline TST (min) & $302 \pm 83$ & $300 \pm 81$ \\
Sleep efficiency (\%) & $73 \pm 18$ & $74 \pm 17$ \\
S1 (\%) & $12 \pm 13$ & $9 \pm 8$ \\
$\mathrm{~S} 2(\%)$ & $62 \pm 16$ & $63 \pm 10$ \\
$\mathrm{~S} 3+4(\%)$ & $10 \pm 8$ & $15 \pm 10$ \\
$\mathrm{REM} \mathrm{( \% )}$ & $13 \pm 6$ & $12 \pm 7$ \\
Arousal I (N/h) & $24 \pm 14$ & $24 \pm 16$ \\
AHI (events/h) & $20 \pm 16$ & $28 \pm 23$ \\
Mean SaO $(\%)$ & $93 \pm 2$ & $93 \pm 3$ \\
Lowest $\mathrm{SaO}_{2}(\%)$ & $80 \pm 10$ & $82 \pm 6$ \\
ESS & $9 \pm 6$ & $11 \pm 6$
\end{tabular}

Data are reported as mean $\pm \mathrm{SD}$. TST = total sleep time; $\mathrm{S} 1=$ sleep stage $1 ; \mathrm{S} 2=$ sleep stage 2; S3 $+4=$ sleep stage 3 plus sleep stage $4 ; \mathrm{REM}=$ rapid eye movement sleep; arousal I = arousal index per hour; AHI = apnea-hypopnea index; $\mathrm{SaO}_{2}=$ arterial oxygen saturation; ESS $=$ Epworth Sleepiness Scale. There were no significant differences between groups (Student unpaired $t$-test). 
Table 3. Demographic, clinical and laboratory findings of patients with congestive heart failure according to gender.

\begin{tabular}{lcc}
\hline & $\begin{array}{c}\text { Male } \\
\mathrm{N}=60(67 \%)\end{array}$ & $\begin{array}{c}\text { Female } \\
\mathrm{N}=29(33 \%)\end{array}$ \\
\hline Age (years) & $57 \pm 12$ & $59 \pm 12$ \\
$\mathrm{BMI}\left(\mathrm{kg} / \mathrm{m}^{2}\right)$ & $26 \pm 4$ & $26 \pm 6$ \\
$\mathrm{SBP}(\mathrm{mmHg})$ & $123 \pm 21$ & $118 \pm 34$ \\
$\mathrm{DBP}(\mathrm{mmHg})$ & $79 \pm 12$ & $77 \pm 15$ \\
$\mathrm{LVEF}(\%)$ & $34 \pm 6$ & $37 \pm 6$ \\
$\mathrm{NYHA}$ functional class & & \\
II & $56 \%$ & $66 \%$ \\
III & $32 \%$ & $24 \%$ \\
IV & $12 \%$ & $10 \%$ \\
Awake arterial blood gases & & \\
Arterial pH & $7.36 \pm 0.45$ & $7.42 \pm 0.03$ \\
$\mathrm{PaCO}(\mathrm{mmHg})$ & $37 \pm 5$ & $36 \pm 5$ \\
$\mathrm{PaO}_{2}(\mathrm{mmHg})$ & $79 \pm 8.5$ & $82 \pm 10$ \\
\hline
\end{tabular}

Data are reported as mean \pm SD or percent. $\mathrm{BMI}=$ body mass index; SBP = systolic blood pressure; $\mathrm{DBP}=$ diastolic blood pressure; $L V E F=$ left ventricular ejection fraction; NYHA = New York Heart Association; $\mathrm{PaCO}_{2}=$ arterial carbon dioxide tension; $\mathrm{PaO}_{2}=$ arterial oxygen tension. There were no significant differences between groups (Student unpaired $t$-test and $Z$ test).

Table 4. Polysomnographic characteristics of congestive heart failure patients according to gender.

\begin{tabular}{lcc}
\hline & Males & Females \\
& $N=60(67 \%)$ & $N=29(33 \%)$ \\
\hline TST (min) & $288 \pm 86$ & $327 \pm 63^{*}$ \\
Sleep onset (min) & $17 \pm 23$ & $15 \pm 19$ \\
Sleep efficiency (\%) & $71 \pm 18$ & $79 \pm 14^{*}$ \\
S1 (\%) & $12 \pm 11$ & $7 \pm 6^{*}$ \\
S2 (\%) & $65 \pm 11$ & $58 \pm 14^{*}$ \\
S3 $+4(\%)$ & $11 \pm 9$ & $19 \pm 8^{*}$ \\
REM (\%) & $12 \pm 7$ & $14 \pm 5$ \\
Arousal I (N/h) & $27 \pm 15$ & $20 \pm 15$ \\
AHI (events/h) & $29 \pm 23$ & $19 \pm 16^{*}$ \\
SaO mean (\%) & $93 \pm 3$ & $94 \pm 2$ \\
SaO 2 lowest (\%) & $82 \pm 7$ & $82 \pm 8$ \\
SDB (\%) & $62 \%$ & $45 \% *$ \\
CSR (\%) & $50 \%$ & $38 \%$ \\
OSA (\%) & $12 \%$ & $7 \%$ \\
CSR awake (\%) & $57 \%$ & $24 \% *$ \\
ESS & $12 \pm 5$ & $9 \pm 6^{*}$ \\
\hline
\end{tabular}

Data are reported as mean \pm SD or percent. TST $=$ total sleep time; $\mathrm{S} 1$ = sleep stage $1 ; \mathrm{S} 2$ = sleep stage $2 ; \mathrm{S} 3+4=$ sleep stage 3 plus sleep stage 4 ; REM = rapid eye movement sleep; arousal I = arousal index per hour; $\mathrm{AHI}=$ apnea-hypopnea index; SDB = sleep-disordered breathing; CSR = Cheyne-Stokes respiration during sleep; OSA = obstructive sleep apnea; CSR awake = Cheyne-Stokes respiration while awake; ESS = Epworth Sleepiness Scale.

${ }^{*} \mathrm{P}<0.05$ compared to males (Student unpaired $t$-test). observed in 33 patients (44\%), of whom 26 (79\%), 2 (6\%), and $5(15 \%)$ had CSR, OSA and no SDB, respectively, during PSG recording. Among the 42 (56\%) patients without CSR while awake, 10 (23\%), 4 (9\%), and 28 (66\%) presented CSR, OSA and no SDB, respectively. Eightyone $(91 \%)$ of all patients were followed up for $25 \pm 10$ months, 8 patients were lost to follow-up either because their telephone number had changed or because they did not answer the call. During that period (September 2000 to March 2004), 22 patients (27\%) died. Eighteen patients $(82 \%)$ died due to cardiac causes and $4(18 \%)$ due to noncardiac causes ( 1 died during gastric surgery, 1 due to pneumonia, and 2 of causes associated with renal failure). Demographic, clinical, and laboratory findings and PSG data of survivors and non-survivors are shown in Tables 5 and 6, respectively. The proportion of absence of SDB, CSR and OSA in survivors versus non-survivors was simi$\operatorname{lar}(45,49$ and $6 \%$ versus 50,40 , and $10 \%$, respectively). Non-survivors had lower LVEF and worse NYHA functional classification, less rapid eye movement (REM) sleep, and a higher proportion of CSR while awake than survivors. The log-rank test showed no differences in mortality between CHF subgroups: idiopathic $(P=0.57)$, ischemic $(P=0.58)$, and Chagas' disease $(P=0.73)$. Mortality was similar for males $(29 \%)$ and females $(31 \% ; P=0.41)$, age $(P=0.2)$, and body mass index $(P=0.8)$. The presence of SDB $(P=0.9)$, the AHI $(P=0.41)$, and the percentage of REM sleep $(P=0.09)$ were not associated with mortality. In contrast, NYHA functional classification $(P<0.001)$ as well as the presence of CSR while awake were associated with higher mortality $(P=0.01$; Figure 1$)$. According to the Cox proportional model, NYHA class IV was significantly associated with mortality (relative risk, $\mathrm{RR}=3.95,95 \% \mathrm{Cl}=$ 1.37-11.38, $P=0.011$ ). The presence of CSR while awake was the only other significant variable, but was of marginal statistical significance in this model $(\mathrm{RR}=2.96,95 \% \mathrm{Cl}=$ 0.94-9.33, $\mathrm{P}=0.064$ ).

\section{Discussion}

The results of the present prospective study agree with previous reports, showing a strikingly high prevalence of SDB in consecutive CHF patients. Our study extends current knowledge by showing that: 1) CHF patients with Chagas' disease, a common condition in the South American population, had a frequency of SDB similar to that observed in other etiologies of CHF; 2) men presented more frequent and more severe SDB than women. These differences were associated with poor sleep architecture in men despite similar cardiac function; 3) we did not demonstrate a significant impact of SDB on mortality. In 
contrast, the presence of CSR while awake was associated with a poor prognosis in univariate analysis and may have a prognostic value.

The prevalence of SDB in the general population is more common in men than women (15 vs $9 \%$, respectively, defined as an AHI >5 events/h of sleep) (26). In agreement with previous studies, we found that, in our population of consecutive CHF patients, $81 \%$ had $\mathrm{AHI}>5$ and $56 \%$ had $\mathrm{AHI}>15$. The present study extends these findings by comparing in a prospective study the characteristics of sleep in men and women with CHF. Women had a significantly lower frequency of SDB as well as a significantly lower overall AHI when compared to men. In addition, women had better sleep quality. These differences cannot be accounted for by demographics or LVEF, which were similar for men and women. Therefore, as is the case for the general population, women with $\mathrm{CHF}$ are protected from SDB.

The reasons for gender differences in the expression of SDB are not completely understood. In the general population, SDB is mainly represented by OSA, and obesity is the major risk factor. Increased fat around the neck, related to an android pattern of adiposity, may contribute to pharyngeal narrowing and collapsibility (27). In addition, the inherent characteristics of pharyngeal tissue and central controller may also differ between genders (28). In CHF patients, SDB is mainly represented by CSR. CSR is the consequence of respiratory instability, characterized by hyperventilation in a crescendo-decrescendo pattern that is interposed by apneas or hypopneas (11). In contrast to OSA, which is restricted to sleep, the CSR pattern of breathing may also be present while awake $(12,13)$ as well as during exercise (14-16). The mechanisms leading to CSR are not completely understood, but low arterial car-

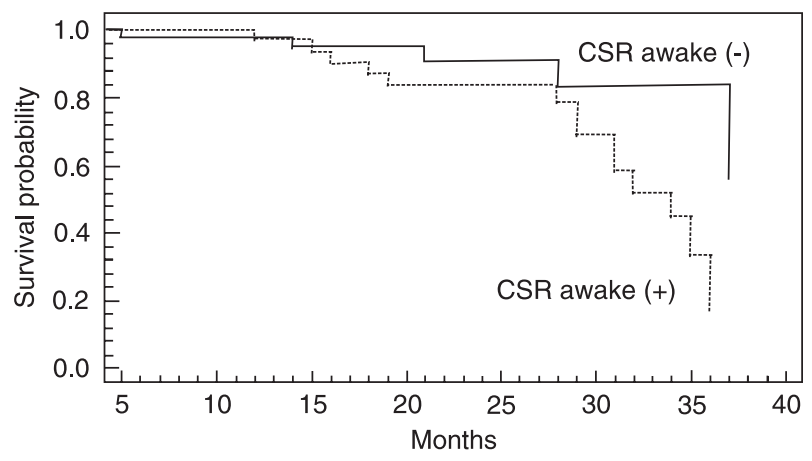

Figure 1. Kaplan-Meier survival curve for patients with CheyneStokes respiration while awake (CSR awake $(+))$ or without (CSR awake (-)). Note that CSR awake (+) patients had a significantly higher time-adjusted mortality rate $(P=0.01$, log-rank test). bon dioxide tension $\left(\mathrm{PaCO}_{2}\right)$ is one of the key factors contributing to respiratory instability (29). Patients with CSR have a low $\mathrm{PaCO}_{2}$ both when awake and asleep (29) and inhalation of small quantities of $\mathrm{CO}_{2}$ abolishes CSR (30). The reasons for hyperventilation and low $\mathrm{PaCO}_{2}$ in patients with $\mathrm{CHF}$ are multiple, and include increased

Table 5. Demographic, clinical, and laboratory findings of surviving and non-surviving patients with congestive heart failure.

Survivors $(\mathrm{N}=58)$ Non-survivors $(\mathrm{N}=23)$

\begin{tabular}{lcc}
\hline Age (years) & $58 \pm 11$ & $56 \pm 14$ \\
Male/female (N; \%) & $33 / 18(65 / 35 \%)$ & $13 / 7(65 / 35 \%)$ \\
BMI (kg/m²) & $26 \pm 6$ & $26 \pm 5$ \\
SBP (mmHg) & $124 \pm 27$ & $113 \pm 22$ \\
DBP (mmHg) & $80 \pm 12$ & $77 \pm 12$ \\
LVEF (\%) & $36.5 \pm 5$ & $31.5 \pm 7^{*}$ \\
NYHA functional class & & \\
II & $69 \%$ & $45 \%$ \\
III & $25 \%$ & $30 \%$ \\
IV & $6 \%$ & $25 \% *$ \\
Awake arterial blood gases & \\
Arterial pH & $7.42 \pm 0.03$ & $7.44 \pm 0.03$ \\
$\mathrm{PaCO}_{2}(\mathrm{mmHg})$ & $37 \pm 4$ & $35 \pm 7$ \\
$\mathrm{PaO}_{2}(\mathrm{mmHg})$ & $79 \pm 8$ & $81 \pm 13$ \\
\end{tabular}

Data are reported as mean \pm SD or percent. BMI = body mass index; SBP = systolic blood pressure; $\mathrm{DBP}=$ diastolic blood pressure; LVEF = left ventricular ejection fraction; NYHA = New York Heart Association; $\mathrm{PaCO}_{2}=$ arterial carbon dioxide tension; $\mathrm{PaO}_{2}=$ arterial oxygen tension.

${ }^{*} \mathrm{P}<0.05$ compared to survivors (Student unpaired $t$-test and $\mathrm{Z}$ test).

Table 6. Polysomnographic data of surviving and non-surviving patients with congestive heart failure.

Survivors $(N=58)$ Non-survivors $(N=23)$

\begin{tabular}{lcc}
\hline TST (min) & $315 \pm 77$ & $297 \pm 89$ \\
Sleep efficiency (\%) & $70 \pm 8$ & $70 \pm 20$ \\
S1 (\%) & $8 \pm 6$ & $10 \pm 10$ \\
S2 (\%) & $62 \pm 13$ & $67 \pm 10$ \\
S3 + 4 (\%) & $14 \pm 10$ & $13 \pm 9$ \\
REM (\%) & $14 \pm 7$ & $9 \pm 6^{*}$ \\
Arousal I (N/h) & $22 \pm 14$ & $26 \pm 17$ \\
AHI (events/h) & $25 \pm 22$ & $25 \pm 22$ \\
CSR awake (\%) & $32 \%$ & $53 \%^{*}$ \\
ESS & $10 \pm 6$ & $11 \pm 6$ \\
\hline
\end{tabular}

Data are reported as mean \pm SD or percent. TST $=$ total sleep time; $\mathrm{S} 1$ = sleep stage $1 ; \mathrm{S} 2=$ sleep stage $2 ; \mathrm{S} 3+4=$ sleep stage 3 plus sleep stage 4 ; REM = rapid eye movement sleep; arousal I = arousal index per hour; $\mathrm{AHI}=$ apnea-hypopnea index; CSR awake $=$ Cheyne-Stokes respiration while awake; $\mathrm{ESS}=$ Epworth Sleepiness Scale.

${ }^{*} \mathrm{P}<0.05$ compared to survivors (Student unpaired $t$-test). 
peripheral and central chemosensitivity as well as pulmonary congestion $(29,31,32)$. Our study agrees with previous reports showing that CHF patients with CSR have a lower awake $\mathrm{PaCO}_{2}$ than patients with similar LVEF but no CSR. The awake $\mathrm{PaCO}_{2}$ was similar in men and women, suggesting that this is not a mechanism explaining gender differences in propensity to SDB. However, differences in the respiratory controller may be present during sleep (33). Central events occur most frequently during the lighter stages of non-REM sleep, particularly after arousals and sleep stage changes. In the general population, males tend to have a more fragmented sleep than females (34). In our CHF population, men had a less stable sleep architecture than women, with a larger number of sleep-wake transitions and shorter slow-wave sleep. Therefore, sleep instability may help explain the increased risk for CSR associated with male gender.

The comparison of sleep patterns in patients with $\mathrm{CHF}$ has been previously reported for a retrospective study by Sin et al. (8). As in Sin's study, we found that SDB is more common in men than in women with CHF. The major difference between Sin's study and ours is that we found a lower prevalence of OSA in our women (14\%) compared to theirs (31\%). One possible explanation for this difference is that there is no clear-cut separation between obstructive and central apneas, and different criteria may have been employed in the two studies. In patients with CHF, OSA and CSR may be difficult to distinguish. Furthermore, patients with CHF may experience a conversion of predominantly obstructive to predominantly central respiratory events over a single night (35). On the other hand, differences between studies may be accounted for by differences in study design. Suspicion of SDB was one criterion for referral to Sin's retrospective study (8). Because OSA is the major cause for sleep laboratory referrals, this may have biased their study sample toward a higher prevalence of OSA. In contrast, our study recruited consecutive patients at the heart failure clinic. Supporting this finding is the fact that the proportion of OSA found in our study is much closer to Javaheri's prospective studies in patients with CHF $(19,23)$.

The results of our study agree with reports $(8,32)$ demonstrating that patients with $\mathrm{CHF}$ have very poor sleep quality, as shown by low sleep efficiency. The poor sleep quality cannot be attributed to SDB alone and may be caused by the underlying disease state. The absence of difference in daytime sleepiness assessed by the Epworth Sleepiness Scale in CHF patients with and without SDB has also been previously reported (36). Therefore, despite the high prevalence of SDB in patients with $\mathrm{CHF}$, even in patients with Chagas' disease, the symptoms of disturbed sleep overlap with the CHF symptoms, making it difficult to recognize SDB based on symptoms alone.

To the best of our knowledge, ours is the first study to investigate sleep in patients with Chagas' disease, an important cause of CHF in Latin America that has unique characteristics (37). For instance, the congestive phenomenon of heart failure in Chagas' disease is usually more intense in the systemic than in the pulmonary circulation (10). Because pulmonary congestion may cause hyperventilation and low $\mathrm{PaCO}_{2}$ (a major cause of respiratory instability, as previously discussed), we initially hypothesized that patients with Chagas' disease would be protected against CSR. However, we demonstrated that the sleep pattern and the prevalence of SDB, including presence of CSR, was similar across CHF etiology, including those patients with Chagas' disease. Our data support the concept that $\mathrm{CHF}$ is the final pathway of several cardiac etiologies. Once CHF is established, it triggers a large number of stereotyped responses that, at this point, are independent of the original etiology.

There is recent evidence that OSA is associated with increased mortality in patient with CHF (7). In contrast, the information on the impact of CSR on mortality is contradictory. In our study, CSR during sleep was not related to overall mortality in $\mathrm{CHF}$, which is in agreement with some $(13,18)$ but in conflict with other reports $(4,5,19)$. Significant variations between studies, including population characteristics, monitoring system, and definitions may account for these contradictory results (18). In contrast to sleep, the studies on the significance of CSR at rest while awake are more consistent, and systematically show a worse prognosis $(13,16)$. Sharing several similarities with CSR while awake, some studies have shown a poor prognostic value of exercise-related periodic breathing $(14,15)$. Exercise-related periodic breathing may correspond to a mild form of CSR, in which cyclic oscillations in ventilation occur. Recently, Corrà et al. (16) suggested that CSR while awake is significantly associated with SDB (AHI $>30$ ), and that their combination represents a crucial prognostic burden in patients with CHF. However, these authors did not monitor sleep but only performed respiratory screening, and it is not clear if the CSR observed during the night occurred during sleep or wakefulness. Our study shares similarities with a report by Andreas et al. (13) who also showed that CSR while awake, but not during sleep, predicted mortality. In the present study we found, in agreement with previous studies (4), that LVEF and NYHA category were predictors of mortality. The observation that CSR while awake did not remain statistically significant in multivariate analysis may be the result of a low statistical power of the study. Alternatively, CSR may just be a 
marker of low cardiac performance illustrated by a general measure represented by the cardiac functional class.

Our study has several potential limitations. First, only $19 \%$ of the patients studied were on $ß$-blockers, a relatively small number when compared with current guidelines (38). There is some evidence that ß-blockers may be associated with a decrease in the prevalence and severity of CSR $(39,40)$. Therefore, the absolute prevalence of CSR must be interpreted with caution. Second, the number of patients with OSA $(N=9)$, women $(N=29)$ as well as patients with Chagas' disease $(\mathrm{N}=25)$ was relatively small and did not permit a separate mortality analysis in these groups. However, this is the first prospective study to show significant gender differences in sleep in groups with similar anthropometric and cardiac function. Finally, the marginal significance of CSR while awake in predicting mortality does not allow definitive conclusions and may also be the result of a limited number of patients evaluated during follow-up.

Our nonselected, consecutive patients with severe $\mathrm{CHF}$ had high prevalence of SDB irrespective of etiology. Men had a higher prevalence of SDB, more severe SDB and worse sleep quality than women. Presence of CSR while awake, but not during sleep, may be a marker of poor prognosis in patients with CHF.

\section{Acknowledgments}

Sincere thanks to Ana Cristina Muriel, Lygia Mattos Silva, and Fabio Hilario for technical assistance.

\section{References}

1. McMurray JJ, Pfeffer MA. The year in heart failure. J Am Coll Cardiol 2004; 44: 2398-2405.

2. Lloyd-Jones DM, Larson MG, Leip EP, Beiser A, D'Agostino $\mathrm{RB}$, Kannel WB, et al. Lifetime risk for developing congestive heart failure: the Framingham Heart Study. Circulation 2002; 106: 3068-3072.

3. Heiat A, Gross CP, Krumholz HM. Representation of the elderly, women, and minorities in heart failure clinical trials. Arch Intern Med 2002; 162: 1682-1688.

4. Hanly PJ, Zuberi-Khokhar NS. Increased mortality associated with Cheyne-Stokes respiration in patients with congestive heart failure. Am J Respir Crit Care Med 1996; 153: 272-276.

5. Lanfranchi PA, Braghiroli A, Bosimini E, Mazzuero G, Colombo R, Donner CF, et al. Prognostic value of nocturnal Cheyne-Stokes respiration in chronic heart failure. Circulation 1999; 99: 1435-1440.

6. Findley LJ, Zwillich CW, Ancoli-Israel S, Kripke D, Tisi G, Moser KM. Cheyne-Stokes breathing during sleep in patients with left ventricular heart failure. South Med J 1985; 78: $11-15$.

7. Wang H, Parker JD, Newton GE, Floras JS, Mak S, Chiu $\mathrm{KL}$, et al. Influence of obstructive sleep apnea on mortality in patients with heart failure. J Am Coll Cardiol 2007; 49: 1625-1631.

8. Sin DD, Fitzgerald F, Parker JD, Newton G, Floras JS, Bradley TD. Risk factors for central and obstructive sleep apnea in 450 men and women with congestive heart failure. Am J Respir Crit Care Med 1999; 160: 1101-1106.

9. Oldenburg O, Lamp B, Faber L, Teschler H, Horstkotte D, Topfer V. Sleep-disordered breathing in patients with symptomatic heart failure: a contemporary study of prevalence in and characteristics of 700 patients. Eur J Heart Fail 2007; 9: 251-257.

10. WHO. Control of Chagas' disease: second report of the WHO Expert Committee. World Health Organ Tech Rep Ser 2002; 905: 1-109.
11. Lorenzi-Filho G, Genta PR, Figueiredo AC, Inoue D. Cheyne-Stokes respiration in patients with congestive heart failure: causes and consequences. Clinics 2005; 60: 333344.

12. Ponikowski P, Anker SD, Chua TP, Francis D, Banasiak W, Poole-Wilson PA, et al. Oscillatory breathing patterns during wakefulness in patients with chronic heart failure: clinical implications and role of augmented peripheral chemosensitivity. Circulation 1999; 100: 2418-2424.

13. Andreas S, Hagenah G, Moller C, Werner GS, Kreuzer H. Cheyne-Stokes respiration and prognosis in congestive heart failure. Am J Cardiol 1996; 78: 1260-1264.

14. Leite JJ, Mansur AJ, de Freitas HF, Chizola PR, Bocchi EA, Terra-Filho $\mathrm{M}$, et al. Periodic breathing during incremental exercise predicts mortality in patients with chronic heart failure evaluated for cardiac transplantation. J Am Coll Cardiol 2003; 41: 2175-2181.

15. Corrà U, Giordano A, Bosimini E, Mezzani A, Piepoli M, Coats AJ, et al. Oscillatory ventilation during exercise in patients with chronic heart failure: clinical correlates and prognostic implications. Chest 2002; 121: 1572-1580.

16. Corrà U, Pistono M, Mezzani A, Braghiroli A, Giordano A, Lanfranchi $P$, et al. Sleep and exertional periodic breathing in chronic heart failure: prognostic importance and interdependence. Circulation 2006; 113: 44-50.

17. Naughton MT, Bradley TD. Sleep apnea in congestive heart failure. Clin Chest Med 1998; 19: 99-113.

18. Roebuck T, Solin P, Kaye DM, Bergin P, Bailey M, Naughton MT. Increased long-term mortality in heart failure due to sleep apnoea is not yet proven. Eur Respir J 2004; 23: 735740.

19. Javaheri S, Shukla R, Zeigler H, Wexler L. Central sleep apnea, right ventricular dysfunction, and low diastolic blood pressure are predictors of mortality in systolic heart failure. J Am Coll Cardiol 2007; 49: 2028-2034.

20. Rechtschaffen A, Kales A. A manual of standardized terminology: Techniques and scoring system for sleep stages of 
human subjects. Los Angeles: UCLA Brain Information Service/Brain Research Institute; 1968.

21. ASDA (American Sleep Disorders Association) Atlas Task Force. EEG arousals: scoring rules and examples. Sleep 1992; 15: 174-184.

22. AASM (American Academy of Sleep Medicine) Task Force. Sleep-related breathing disorders in adults: recommendations for syndrome definition and measurement techniques in clinical research. Sleep 1999; 22: 667-689.

23. Javaheri S. Sleep disorders in systolic heart failure: a prospective study of 100 male patients. The final report. Int $J$ Cardiol 2006; 106: 21-28.

24. Lanfranchi PA, Somers VK, Braghiroli A, Corra U, Eleuteri E, Giannuzzi P. Central sleep apnea in left ventricular dysfunction: prevalence and implications for arrhythmic risk. Circulation 2003; 107: 727-732.

25. Johns MW. A new method for measuring daytime sleepiness: the Epworth Sleepiness Scale. Sleep 1991; 14: 540545.

26. Young T, Palta M, Dempsey J, Skatrud J, Weber S, Badr S. The occurrence of sleep-disordered breathing among middle-aged adults. N Engl J Med 1993; 328: 1230-1235.

27. Horner RL, Mohiaddin RH, Lowell DG, Shea SA, Burman $E D$, Longmore $D B$, et al. Sites and sizes of fat deposits around the pharynx in obese patients with obstructive sleep apnoea and weight matched controls. Eur Respir J 1989; 2: 613-622.

28. Pillar G, Malhotra A, Fogel R, Beauregard J, Schnall R, White DP. Airway mechanics and ventilation in response to resistive loading during sleep: influence of gender. $A m \mathrm{~J}$ Respir Crit Care Med 2000; 162: 1627-1632.

29. Javaheri S. A mechanism of central sleep apnea in patients with heart failure. N Engl J Med 1999; 341: 949-954.

30. Lorenzi-Filho G, Rankin F, Bies I, Douglas BT. Effects of inhaled carbon dioxide and oxygen on Cheyne-Stokes respiration in patients with heart failure. Am J Respir Crit Care Med 1999; 159: 1490-1498.
31. Solin P, Roebuck T, Johns DP, Walters EH, Naughton MT. Peripheral and central ventilatory responses in central sleep apnea with and without congestive heart failure. Am J Respir Crit Care Med 2000; 162: 2194-2200.

32. Hanly PJ, Millar TW, Steljes DG, Baert R, Frais MA, Kryger $\mathrm{MH}$. Respiration and abnormal sleep in patients with congestive heart failure. Chest 1989; 96: 480-488.

33. Zhou XS, Shahabuddin S, Zahn BR, Babcock MA, Badr MS. Effect of gender on the development of hypocapnic apnea/hypopnea during NREM sleep. J Appl Physiol 2000; 89: 192-199.

34. Hume KI, Van F, Watson A. A field study of age and gender differences in habitual adult sleep. J Sleep Res 1998; 7: 8594.

35. Tkacova R, Niroumand M, Lorenzi-Filho G, Bradley TD. Overnight shift from obstructive to central apneas in patients with heart failure: role of $\mathrm{PCO}_{2}$ and circulatory delay. Circulation 2001; 103: 238-243.

36. Hanly P, Zuberi-Khokhar N. Daytime sleepiness in patients with congestive heart failure and Cheyne-Stokes respiration. Chest 1995; 107: 952-958.

37. Bestetti RB, Muccillo G. Clinical course of Chagas' heart disease: a comparison with dilated cardiomyopathy. Int $J$ Cardiol 1997; 60: 187-193.

38. Packer M, Cohn JN on behalf of the Steering Committee and Membership of the Advisory Council to Improve Outcomes Nationwide in Heart Failure. Consensus recommendations for the management of chronic heart failure, II: management of heart failure: approaches to the prevention of heart failure. Am J Cardiol 1999; 83: 9A-38A.

39. Tamura A, Kawano Y, Naono S, Kotoku M, Kadota J. Relationship between beta-blocker treatment and the severity of central sleep apnea in chronic heart failure. Chest 2007; 131: 130-135.

40. Kohnlein T, Welte T. Does beta-blocker treatment influence central sleep apnoea? Respir Med 2007; 101: 850-853. 